\title{
Rho-kinase and Insulin Signaling
}

\author{
Young-Bum Kim \\ Division of Endocrinology, Diabetes and Metabolism, \\ Beth Israel Deaconess Medical Center and Harvard Medical School
}

\begin{abstract}
Insulin's ability to acutely stimulate glucose uptake and metabolism in peripheral tissues is essential for normal glucose homeostasis. Resistance to this insulin effect is a major pathogenic feature of type 2 diabetes and obesity. Although many of the proximal steps in insulin signaling have been identified, the molecular mechanisms underlying insulin resistance under various metabolic states are still unclear. Recent study suggests that Rho-kinase is an important mediator of insulin signaling and glucose homeostasis. Specifically, Rho-kinase directly controls insulin receptor substrate-1, which plays an important role in regulating insulin action. Inhibition of Rho-kinase function results in a decreased insulin response, leading to insulin resistance. Thus, Rho-kinase is identified as a novel regulator of insulin action and glucose homeostasis, and a potential target for new diabetes drugs. (J Kor Diabetes Assoc 30:237 245, 2006)
\end{abstract}

\section{Introduction}

Impairment of the normal response to insulin (insulin resistance) is a major risk factor for type 2 diabetes and contributes to the morbidity of obesity. Insulin resistance even without overt hyperglycemia is associated with other common disorders, including hypertension, dyslipidemia, cardiovascular disease and cardiac death, as well as polycystic ovarian disease ${ }^{1}$. When insulin resistance cannot be compensated for by increased insulin secretion, frank hyperglycemia or diabetes occurs ${ }^{2}$. In insulin-resistant states, there are defects in multiple actions of insulin, including: 1) impaired stimulation of glucose uptake in skeletal muscle and adipocytes, 2) impaired inhibition of glucose production from the liver, and 3) alterations in lipid metabolism causing dyslipidemia ${ }^{1)}$. The mechanisms underlying insulin resistance remain largely unknown although they are thought to result, at least in part, from impaired insulin-stimulated signal transduction.

Insulin action is mediated by a cascade of tyrosyl phosphorylation events, initiated by insulin binding to its cell surface receptor (an intrinsic receptor tyrosine kinase), followed by receptor autophosphorylation and activation of insulin receptor (IR) kinase activity ${ }^{3)}$. The kinase activity of IR is essential for downstream activation of virtually all of insulin's metabolic and growth-promoting effects $^{4}$. This has been shown using cells from patients with naturally occurring IR mutations as well as by site-directed mutagenesis of the $\mathrm{IR}^{5)}$. The activated IR kinase phosphorylates insulin receptor substrates (IRSs) on multiple tyrosine residues. Phosphotyrosine residues on IRSs act as docking sites for many src homology-2 (SH2) domain-containing proteins, including the p85 regulatory subunit of phosphoinositide 3-kinase (PI 3-kinase). Upon binding to IRS proteins, PI 3-kinase is activated, leading to a number of insulin's metabolic effects. In muscle and adipocytes, activation of PI 3-kinase is necessary, although not fully sufficient, for stimulation of glucose transport $^{6,7)}$. Rho-kinase, a downstream target for Rho, has been shown to participate in the insulin signaling network by interacting with IRS-1 ${ }^{8,9)}$. We discuss here the role of the Rho-kinase pathway in IRS-1-mediated insulin signaling and glucose metabolism.

\section{Regulation of small G-proteins}

Small G-proteins of the Rho family are monomeric 
A.

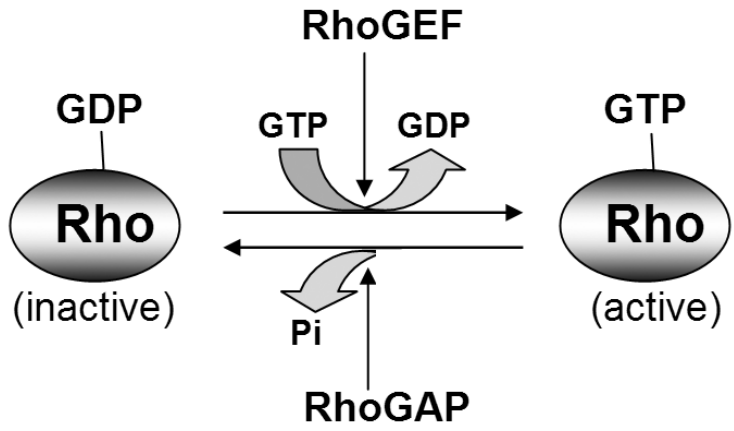

B. GTP-Rho $\rightarrow-$

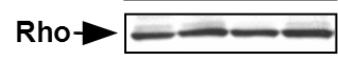

Insulin: - + - +

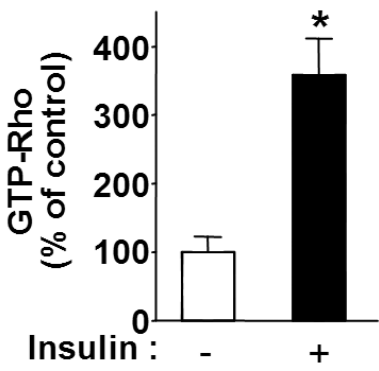

Fig. 1. Regulation of Rho.

\begin{tabular}{|c|c|c|c|c|c|}
\hline \multirow[b]{2}{*}{ ROK $\alpha$} & 76 & 338 & \multicolumn{3}{|r|}{1354} \\
\hline & $\mathrm{N}-\mathrm{C}$ & & Coiled-coil region & RBD & PHCysrich PH - C \\
\hline & 76 & 354 & & & \\
\hline OK & $\mathbf{N}=$ & t & Coiled-coil region & RBD & PH:Cysrich PH \\
\hline
\end{tabular}

Fig. 2. The structure of Rho-kinase.

guanosine nucleotide-binding proteins of $25 \sim 35 \mathrm{kDa}$. Rho proteins are inactive when bound to GDP and activation occurs when GDP is released and replaced by GTP. This cycling is largely regulated by two classes of proteins, guanine nucleotide exchange factors (GEFs) and GTPase activating proteins (GAPs) ${ }^{10,11)}$ (Fig. 1). RhoGEFs function to promote the conversion of inactive Rho to active Rho, while RhoGAPs stimulate the intrinsic GTP hydrolysis activity of Rho protein, thereby converting it to a GDP-bound, inactive form ${ }^{10,11)}$ (Fig. 1A). Activation of Rho by various agonists requires translocation of inactive cytosolic Rho to membranes. Thus, appearance of Rho in the membrane fraction is indicative of Rho activation ${ }^{12,13)}$. In isolated adipocytes, insulin stimulates Rho translocation to the membrane by a PI 3-kinase dependent mechanism ${ }^{13,14)}$. Recent findings also demonstrate that insulin activates GTP-Rho activity in skeletal muscle in vivo ${ }^{15)}$ (Fig. 1B), suggesting that Rho signaling is involved in the insulin signaling pathway.

\section{Rho-kinase structure}

Rho-kinase is a Ser/Thr protein kinase identified as a GTP-Rho-binding protein ${ }^{16)}$. There are two isoforms of Rho-kinase, ROKa (also known as ROCK-2) ${ }^{17,18)}$ and
$\operatorname{ROK} \beta$ (also known as ROCK-1) ${ }^{18,19)}$. Rho-kinase has a kinase domain at its $\mathrm{N}$-terminal domain, a coiled-coil domain in its middle portion, and a putative pleckstrin-homology $(\mathrm{PH})$ domain at its C-terminal domain split by the insertion of a Cys-rich region. The Rho binding domain (RBD) of Rho-kinase is in the C-terminal portion of the coiled-coil domain ${ }^{16)}$ (Fig. 2). The lack of the C-terminal portion in Rho-kinase, either $\mathrm{PH}$ or $\mathrm{PH}$ with coiled-coil domains, makes Rho-kinase constitutively active. In contrast, the kinase activity deficient form, or the C-terminal fragments that lack the kinase domain, serves as the dominant-negative form of Rho-kinase in cells ${ }^{16}$. The amino acid sequences of the two Rho-kinase isoforms have $65 \%$ overall identity, and the proteins show the highest similarity to their kinase domains (92\% identity).

\section{Rho-kinase tissue expression and subcellular localization}

Despite the striking similarity of the protein sequences of the two ROCK isoforms, significant differences regarding their respective tissue distribution have been reported, which indicates distinct functions of each isoform in vivo. ROCK-2 is preferentially expressed in 
brain, whereas ROCK-1 shows the highest expression levels in non-neuronal tissues, including heart, lung and skeletal muscles. Both ROCK isoforms are also expressed in the adipose tissues of rodents. ROCK- 2 in bovine brain is mainly expressed in the pyramidal neurons of the hippocampus and cerebral cortex, as well as in the Purkinje cells of the cerebellum ${ }^{20}$. Interestingly, during postnatal development of the mouse brain, ROCK-2 expression levels gradually increased ${ }^{21)}$. These findings indicate that the proteins might have a special role in these cell/tissue types. Localization of ROCK-2 by immunofluorescence technique has shown that the protein is distributed mainly in the cytoplasma ${ }^{16,17)}$. This is supported by cell-fractionation studies showing that the main portion of ROCK-2 is in the cytosolic fraction ${ }^{17)}$. A small amount of ROCK-2 is also founded in the membrane fraction ${ }^{16)}$. Indeed, ROCK-2 translocates from the cell cytosol to plasma membrane when active RhoA is overexpressed $^{16,17)}$ or after stimulation of cells with factors that activate $\mathrm{Rho}^{22,23)}$. The determination of ROCK-1 localization has proven to be more difficult, but a recent report has indicated that it might colocalize with centrosomes $^{24)}$.

\section{Regulation of Rho-kinase activity}

Rho-kinase activity is enhanced by binding with RhoGTP through a Rho-binding domain ${ }^{16}$. In the inactive form, the $\mathrm{PH}$ domain and the Rho binding domain of Rho-kinase bind to the amino-terminal region of the protein, which forms an autoinhibitory loop ${ }^{25)}$. Once GTP-bound Rho binds to the Rho binding domain of Rho-kinase, this results in an open confirmation of the kinase and increases catalytic activity. In addition, intracellular second messengers such as arachidonic acid and sphingosylphosphorylcholine, can activate Rho-kinase independently of $\mathrm{Rho}^{26)}$. Interestingly, ROCK-1, but not ROCK-2, can be activated by caspase-3-mediated cleavage near the carboxyl terminus ${ }^{27)}$. Moreover, recent studies demonstrate that Rho-kinase activity increases in response to insulin in insulin-target tissue of mice ${ }^{15)}$.

\section{Rho-kinase function}

Rho-kinase has been implicated in a variety of cellular functions, including actin cytoskeleton, the assembly of myosin-actin filaments and cell contractility ${ }^{28)}$. Inhibition of Rho-kinase activity by a chemical inhibitor or expression of dominant negative Rho-kinase causes loss of actin stress fibers and focal adhesion complexes ${ }^{29)}$, whereas expression of wild type Rho-kinase induces the formation and contractility of actin stress fibers in several cell types ${ }^{29,30)}$. This is thought to be mediated both by phosphorylating the myosin light chain and by phosphorylating the myosin-binding subunit of myosin phosphatase $^{31,32)}$. Thus Rho-kinase regulates cell polarity and migration through cellular contraction and focal adhesions. Treatment of a hypertensive animal with the Rho-kinase inhibitor results in decreased blood pressure ${ }^{33)}$, suggesting that the Rho-kinase inhibitor is a potential therapeutic target for many diseases that result from abnormally high smooth muscle contraction. Recently, Rho-kinase has been linked to the control of cell size by enhancing IGF-induced cAMP response element binding protein (CREB) phosphorylation ${ }^{34)}$. Furthermore, Rho-kinase is identified as an essential modulator of IGF-1 signals that are involved in the regulation of $\operatorname{adipogenesis}^{35)}$.

\section{The potential role of Rho/Rho-kinase in glucose transport}

Rho proteins play an important role in many cellular processes, including signal transduction, vesicle trafficking from intracellular organelles, and cytoskeletal organization ${ }^{36,37)}$. All of these processes are potentially involved in insulin-stimulated glucose transport in adipocytes and muscle cells ${ }^{38-40)}$. In fact, another small G-protein, TC10 and cdc42, is involved in the regulation of insulin-stimulated Glut4 translocation via a PI-3-kinase independent pathway ${ }^{41)}$. Insulin-stimulated glucose transport is decreased by treatment with $\mathrm{C} 3$ transferase, a Rho inhibitor, and is increased by treatment with GTP $y$, an activator of Rho, in isolated adipocytes ${ }^{13,14)}$. Overexpression of a wild type Rho stimulates glucose transport while a dominant negative form of Rho inhibits insulin-induced glucose transport in 3T3-L1 adipocytes and isolated adipocytes ${ }^{14)}$. In addition, GTP 8 S activates membrane-associated PI 3-kinase in isolated adipocytes but this activation is blocked by a Rho inhibitor. Rho-kinase, one of the major Rho targets, is activated by binding with $\mathrm{Rho}^{42,43)}$. Therefore, it is possible that Rho 


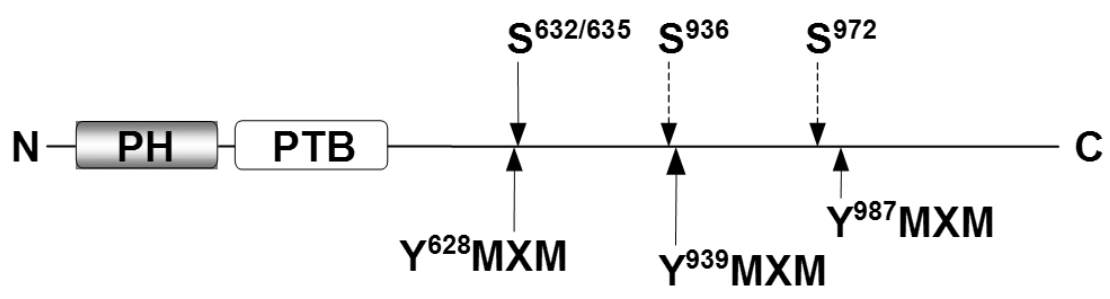

Fig. 3. Schematic diagram of IRS-1 serine phosphorylation sites by Rho-kinase. The relative positions of the pleckstrin homology (PH) and phosphotyrosine binding (PTB) domains are indicated.

may require Rho-kinase activation to mediate its effects on insulin stimulation of glucose transport. Indeed, our recent findings demonstrate that inhibition of Rho-kinase decreases insulin-stimulated glucose transport in 3T3-L1 adipocytes and L6 muscle cells ${ }^{15)}$. In addition, suppression of endogenous Rho-kinase expression by small interfering RNA causes a significant decrease in insulin-induced glucose transport in 3T3-L1 adipocytes. These effects are most likely due to decreased Glut4 translocation ${ }^{15)}$. Collectively, these data support the hypothesis that the Rho/Rho-kinase pathway is necessary for insulin's metabolic action on glucose transport.

\section{Does Rho-kinase regulate insulin signaling?}

Studies suggest that Rho-kinase may play an important role in insulin signaling via a direct interaction with IRS- $1^{8,9)}$. However, it is unclear how Rho-kinase regulates insulin-stimulated glucose metabolism via IRS-1, and the molecular mechanism(s) by which Rho-kinase regulates insulin signaling have not been established. A recent study demonstrates that inhibition of Rho-kinase decreases insulin-stimulated IRS-1-associated PI 3-kinase activity in adipocytes and myotubes ${ }^{15)}$. This is mainly due to decreased tyrosine phosphorylation of the YXXM motif in IRS-1, which can lead to reduced interaction of IRS-1 with the p85 subunit of PI 3-kinase ${ }^{15)}$. Indeed, insulin-stimulated IRS-1 binding to the p85 regulatory subunit of PI 3-kinase is impaired in adipocytes expressing dominant negative Rho-kinase ${ }^{15}$. However, some studies indicate that Rho activation by the expression of constitutively active Rho or absence of Rho-inhibitory protein can impair the early steps of insulin/IGF signaling, including IRS-1 tyrosine phosphorylation in vascular smooth muscle cells ${ }^{9)}$ and fibroblasts derived from p190B RhoGAP null mice ${ }^{34)}$.
Since Rho has multiple target molecules including rhophilin, rhotekin, citron, and protein kinase $\mathrm{N}^{44-47)}$, it is unclear whether an inhibitory role of Rho-kinase on insulin signaling occurs through Rho-kinase itself or other downstream mediators. In this regard, it seems unlikely that the alteration in Rho signaling can simply explain all aspects of downstream pathways. This is supported by the fact that insulin activates MAPK in Xenopus oocytes through a Rho-kinase pathway that is independent of an upstream Rho pathway ${ }^{48)}$.

\section{Rho-kinase and IRS-1 serine phosphorylation}

It is clear that serine phosphorylation of IRS-1 protein in response to insulin has a dual role either to enhance or inhibit insulin signaling ${ }^{49)}$. IRS-1 contains more than 100 potential serine phosphorylation sites ${ }^{3)}$ which are recognized by many kinases, including $\mathrm{JNK}^{50)}$, PKCs ${ }^{51-54)}$, $\mathrm{mTOR}^{55)}, \mathrm{MAPK}^{56)}$ and $\mathrm{AMPK}^{57)}$. It is likely that the IRS-1 function is regulated in several ways, including the interaction between specific serine kinases and IRS-1, regulation of the associated kinase activities, and the selection of specific phosphorylation sites. Therefore, identifying the serine kinase that phosphorylates IRS-1 specific serine residues is important in understanding the mechanism behind the dysregulation of insulin action in altered metabolic states. A recent study finds that Rho-kinase directly phosphorylates serine 632/635, 936 and 972 on IRS-1 in vitro ${ }^{15)}$. Interestingly, serine $632 / 635$ is only four amino acids from tyrosine 628, serine 936 is only two amino acids from tyrosine 939 and serine 972 residue is close to tyrosine 987 (Fig. 3), all of which are in the YMXM motif that is involved in the binding of p85 regulatory subunit of PI 3-kinase ${ }^{49)}$. Replacing these serines with alanine causes a significant inhibition of insulin-stimulated tyrosine phosphorylation of IRS-1 and 


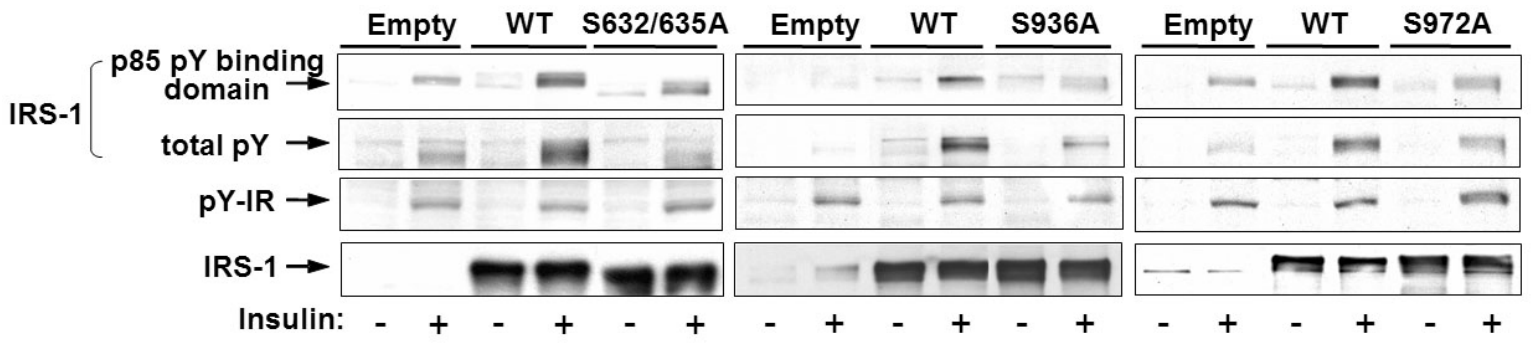

Fig. 4. Effects of IRS-1 serine phosphorylation on insulin signaling in $\mathrm{CHO}_{\mathrm{IR}}$ cells. $\mathrm{CHO}_{\mathrm{IR}}$ cells were transfected with a WT-IRS-1 or a S632/635A-IRS-1 or a S936A-IRS-1 or a S972A-IRS-1cDNA. After 48 hours, cells were stimulated with insulin $(10 \mathrm{nM})$ for 15 minutes. Cell lysates were subjected to immunoprecipitation with an IRS-1 antibody. p85 and IRS-1 were visualized by immunoblotting with a p85 antibody or an IRS-1 antibody. IRS-1 and IR were visualized by immunoblotting with a phosphotyrosine, a phospho-specific IRS-1 or a total IRS-1 antibody.

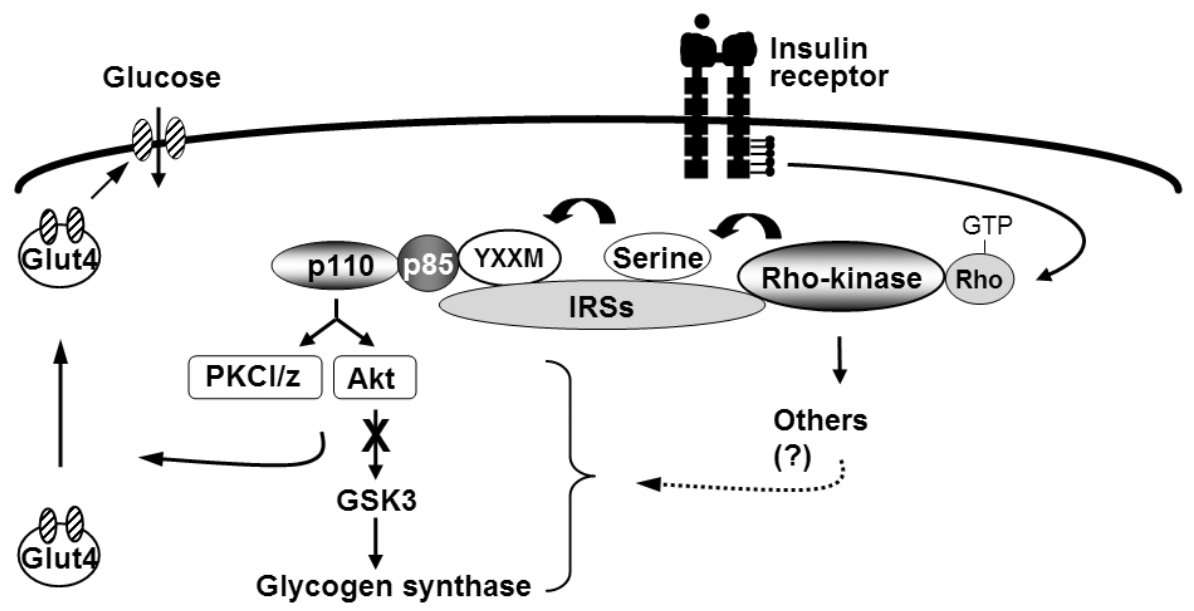

Fig. 5. A hypothetical mechanism by which Rho-kinase enhances insulin signaling and glucose metabolism. Upon insulin stimulation, Rho-kinase is activated and rapidly phosphorylates IRS-1 on serine residues, which in turn increases the ability of IRS-1 to activate PI 3-kinase, leading to enhanced glucose transport activity and other downstream pathways.

insulin-stimulated PI 3-kinase activity associated with IRS-1 (Fig. 4, YBK unpublished data). Thus, it is hypothesized that these serine phosphorylations positively regulate insulin action by facilitating the interaction of IRS-1 with the p85 subunit, leading to increased PI 3-kinase activity (Fig. 4).

Of note, some studies indicate that IRS-1 serine $632 / 635$ phosphorylation is negatively regulated via an mTOR-p70S6K-dependent pathway ${ }^{55,58)}$. A possible explanation for this discrepancy is that these sites in IRS-1 could have a dual role in serving as either a positive or negative modulator of insulin signaling under certain conditions. In fact, phosphorylation of serine 789 in IRS-1 can enhance insulin signaling in AICAR treated myotubes $^{57)}$ while also attenuating insulin signaling in the liver of insulin-resistant animals ${ }^{59)}$.
Role of IRS-1 serine 632/635 in glucose transport

Given that serine 632/635 residue is adjacent to the YXXM motif domain in IRS-1 ${ }^{60)}$, it is conceivable that the reduction in serine phosphorylation of IRS-1 could inhibit a conformational change in the phosphotyrosine -binding domain that enhances its affinity for PI 3-kinase. As a result, the ability of IRS-1 to bind to the p85 subunit is diminished, leading to reduced glucose transport. In fact, expression of S632/635A-IRS-1 inactive mutant significantly decreases insulin-stimulated glucose transport in 3T3-L1 adipocytes (YBK, unpublished data). Concurrently, insulin-induced PI 3-kinase activity associated with IRS-1 is decreased in these cells. These data suggest that IRS-1 serine 632/635 phosphorylation is required for Rho-kinase-mediated glucose transport, establishing a novel signaling mechanism for the 
regulation of glucose transport and insulin signaling. However, these data cannot completely rule out the possibility that some Rho-kinase effects on insulin-stimulated IRS-1 serine phosphorylation might be mediated by other mechanisms, e.g., actin cytoskeleton.

\section{In vivo role of Rho-kinase in glucose metabolism}

It is important to know that Rho-kinase activation contributes to insulin resistance in vivo. Some data demonstrate that long-term Rho-kinase inhibition by 4 weeks treatment with fasudil (a Rho-kinase inhibitor) not only decreased blood pressure but improved glucose tolerance in Zucker obese rats ${ }^{61)}$. However, a recent study finds that treating normal mice with the Rho-kinase inhibitor causes insulin resistance in vivo by reducing insulin-mediated glucose uptake in skeletal muscle and whole body ${ }^{15)}$. Furthermore, our preliminary data show that insulin-stimulated Rho-kinase activity is impaired in the skeletal muscle of insulin-resistant mice with obesity and diabetes (YBK, unpublished data). Therefore, the exact role of Rho-kinase in glucose metabolism in vivo is unclear at this time.

\section{Perspectives}

Recent experiments have defined new functions of Rho-kinase in regulating insulin signaling and glucose transport in cultured cell lines. However, the physiological role of Rho-kinase in glucose homeostasis and insulin action in vivo has not been fully addressed. It is therefore important to investigate whether Rho-kinase activity is altered in vivo in insulin-target tissues under various pathophysiological conditions, and whether such alteration is related to changes in insulin action or sensitivity. In addition, to address the causal relationship between Rho-kinase activity and insulin action (and other phenotypes) in vivo, it is also important to examine the effects of selective Rho-kinase overexpression/deficiency in adipocytes and muscle on whole-body insulin sensitivity, glucose homeostasis, and leanness. Combined, these future studies will identify a novel role/mechanism of the Rho-kinase pathway in the regulation of insulin signaling in insulin-target tissues, which is critical for whole body insulin sensitivity and glucose homeostasis.
Ultimately, these findings may help identify a novel anti-diabetes/obesity drug-target.

\section{Reference}

1. DeFronzo RA: Pathogenesis of type 2 diabetes: metabolic and molecular implications for identifying diabetes. Diabetes Rev 5:177-269, 1997

2. Kahn BB: Type 2 diabetes: when insulin secretion fails to compensate for insulin resistance. Cell 92:593-6, 1998

3. White MF: The insulin signalling system and the IRS proteins. Diabetologia 40 Suppl 2:S2-17, 1997

4. Goldstein BJ, Ahmad F, Ding W, Li PM, Zhang WR: Regulation of the insulin signalling pathway by cellular protein- tyrosine phosphatases. Mol Cell Biochem 182:91-9, 1998

5. Cheatham B, Kahn CR: Insulin action and the insulin signalling network. Endocrin Rev 16:117-42, 1995

6. Frevert EU, Kahn BB: Differential effects of constitutively active phosphatidylinositol 3-kinase on glucose transport, glycogen synthase activity, and DNA synthesis in 3T3-L1 adipocytes. Mol Cell Biol 17:190-8, 1997

7. Holman GD, Kasuga M: From receptor to transporter: insulin signalling to glucose transport. Diabetologia 40:991-1003, 1997

8. Farah S, Agazie Y, Ohan N, Ngsee JK, Liu XJ: $A$ rho-associated protein kinase, ROKalpha, binds insulin receptor substrate-1 and modulates insulin signaling. J Biol Chem 273:4740-6, 1998

9. Begum N, Sandu OA, Ito M, Lohmann SM, Smolenski A: Active Rho kinase (ROK-alpha) associates with insulin receptor substrate-1 and inhibits insulin signaling in vascular smooth muscle cells. J Biol Chem 277:6214-22., 2002

10. Symons M, Settleman J: Rho family GTPases: more than simple switches. Trends Cell Biol 10:415-9, 2000

11. Takai Y, Sasaki T, Matozaki T: Small GTP-binding proteins. Physiol Rev 81:153-208, 2001

12. Gong MC, Fujihara H, Somlyo AV, Somlyo AP: Translocation of rhoA associated with $\mathrm{Ca} 2+$ sensitization of smooth muscle. $J$ Biol Chem 272:10704-9, 1997

13. Karnam P, Standaert ML, Galloway L, Farese RV: Activation and translocation of Rho (and ADP 
ribosylation factor) by insulin in rat adipocytes. Apparent involvement of phosphatidylinositol 3-kinase. J Biol Chem 272:6136-40, 1997

14. Standaert M, Bandyopadhyay G, Galloway L, Ono Y, Mukai $\mathrm{H}$, Farese $\mathrm{R}$ : Comparative effects of GTPgammaS and insulin on the activation of Rho, phosphatidylinositol 3-kinase, and protein kinase $N$ in rat adipocytes. Relationship to glucose transport. J Biol Chem 273:7470-7, 1998

15. Furukawa N, Ongusaha P, Jahng WJ, Araki K, Choi CS, Kim HJ, Lee YH, Kaibuchi K, Kahn BB, Masuzaki H, Kim JK, Lee SW, Kim YB: Role of Rho-kinase in regulation of insulin action and glucose homeostasis. Cell Metabolism 2:119-29, 2005

16. Matsui $\mathrm{T}$, Amano $\mathrm{M}$, Yamamoto $\mathrm{T}$, Chihara $\mathrm{K}$, Nakafuku M, Ito M, Nakano T, Okawa K, Iwamatsu A, Kaibuchi K: Rho-associated kinase, a novel serine/threonine kinase, as a putative target for small GTP binding protein Rho. Embo J 15:2208-16, 1996

17. Leung T, Manser E, Tan L, Lim L: A novel serine/threonine kinase binding the Ras-related RhoA GTPase which translocates the kinase to peripheral membranes. J Biol Chem 270:29051-4, 1995

18. Nakagawa O, Fujisawa K, Ishizaki T, Saito Y, Nakao $\mathrm{K}$, Narumiya S: ROCK-I and ROCK-II, two isoforms of Rho-associated coiled-coil forming protein serine/threonine kinase in mice. FEBS Lett 392:189-93, 1996

19. Ishizaki T, Maekawa $M$, Fujisawa $K$, Okawa $K$, Iwamatsu A, Fujita A, Watanabe N, Saito Y, Kakizuka A, Morii N, Narumiya S: The small GTP-binding protein Rho binds to and activates a $160 \mathrm{kDa}$ Ser/Thr protein kinase homologous to myotonic dystrophy kinase. Embo J 15:1885-93, 1996

20. Hashimoto R, Nakamura Y, Kosako H, Amano M, Kaibuchi K, Inagaki M, Takeda M: Distribution of Rho-kinase in the bovine brain. Biochem Biophys Res Commun 263:575-9, 1999

21. Komagome R, Kimura K, Saito M: Postnatal changes in Rho and Rho-related proteins in the mouse brain. Jpn J Vet Res 47:127-33, 2000

22. Sin WC, Chen XQ, Leung T, Lim L: RhoA-binding kinase alpha translocation is facilitated by the collapse of the vimentin intermediate filament network. Mol Cell Biol 18:6325-39, 1998

23. Royal I, Lamarche-Vane N, Lamorte L, Kaibuchi K,
Park M: Activation of cdc42, rac, PAK, and rho-kinase in response to hepatocyte growth factor differentially regulates epithelial cell colony spreading and dissociation. Mol Biol Cell 11:1709-25, 2000

24. Chevrier V, Piel M, Collomb N, Saoudi Y, Frank R, Paintrand M, Narumiya S, Bornens M, Job D: The Rho-associated protein kinase p160ROCK is required for centrosome positioning. J Cell Biol 157:807-17, 2002

25. Amano M, Chihara $\mathrm{K}$, Nakamura $\mathrm{N}$, Kaneko $\mathrm{T}$, Matsuura Y, Kaibuchi K: The $\mathrm{COOH}$ terminus of Rho-kinase negatively regulates rho-kinase activity. $J$ Biol Chem 274:32418-24, 1999

26. Feng J, Ito M, Kureishi Y, Ichikawa K, Amano M, Isaka $\mathrm{N}$, Okawa $\mathrm{K}$, Iwamatsu A, Kaibuchi $\mathrm{K}$, Hartshorne DJ, Nakano T: Rho-associated kinase of chicken gizzard smooth muscle. J Biol Chem 274:3744-52, 1999

27. Sebbagh M, Renvoize C, Hamelin J, Riche N, Bertoglio J, Breard J: Caspase-3-mediated cleavage of ROCK I induces MLC phosphorylation and apoptotic membrane blebbing. Nat Cell Biol 3:346-52, 2001

28. Fukata Y, Amano M, Kaibuchi K: Rho-Rho-kinase pathway in smooth muscle contraction and cytoskeletal reorganization of non-muscle cells. Trends Pharmacol Sci 22:32-9, 2001

29. Amano M, Chihara K, Kimura K, Fukata Y, Nakamura N, Matsuura Y, Kaibuchi K: Formation of actin stress fibers and focal adhesions enhanced by Rho- kinase. Science 275:1308-11, 1997

30. Leung $\mathrm{T}$, Chen XQ, Manser E, Lim L: The $p 160$ RhoA-binding kinase ROK alpha is a member of a kinase family and is involved in the reorganization of the cytoskeleton. Mol Cell Biol 16:5313-27, 1996

31. Kimura K, Ito M, Amano M, Chihara K, Fukata Y, Nakafuku M, Yamamori B, Feng J, Nakano T, Okawa K, Iwamatsu A, Kaibuchi K: Regulation of myosin phosphatase by Rho and Rho-associated kinase (Rho-kinase). Science 273:245-8, 1996

32. Feng J, Ito M, Ichikawa K, Isaka N, Nishikawa M, Hartshorne DJ, Nakano T: Inhibitory phosphorylation site for Rho-associated kinase on smooth muscle myosin phosphatase. J Biol Chem 274:37385-90, 1999

33. Uehata M, Ishizaki $\mathrm{T}$, Satoh $\mathrm{H}$, Ono $\mathrm{T}$, Kawahara $\mathrm{T}$, Morishita T, Tamakawa H, Yamagami K, Inui J, Maekawa M, Narumiya S: Calcium sensitization of 
smooth muscle mediated by a Rho-associated protein kinase in hypertension. Nature 389:990-4, 1997

34. Sordella R, Classon M, Hu KQ, Matheson SF, Brouns MR, Fine B, Zhang L, Takami H, Yamada Y, Settleman J: Modulation of CREB activity by the Rho GTPase regulates cell and organism size during mouse embryonic development. Dev Cell 2:553-65, 2002

35. Sordella R, Jiang W, Chen GC, Curto M, Settleman J: Modulation of Rho GTPase Signaling Regulates a Switch between Adipogenesis and Myogenesis. Cell 113:147-58, 2003

36. Van Aelst L, D'Souza-Schorey C: Rho GTPases and signaling networks. Genes Dev 11:2295-322, 1997

37. Kaibuchi K, Kuroda S, Amano M: Regulation of the cytoskeleton and cell adhesion by the Rho family GTPases in mammalian cells. Annu Rev Biochem 68:459-86, 1999

38. Omata W, Shibata H, Li L, Takata K, Kojima I: Actin filaments play a critical role in insulin-induced exocytotic recruitment but not in endocytosis of GLUT4 in isolated rat adipocytes. Biochem $J 346 \mathrm{Pt}$ 2:321-8, 2000

39. Patki V, Buxton J, Chawla A, Lifshitz L, Fogarty K, Carrington W, Tuft R, Corvera S: Insulin action on GLUT4 traffic visualized in single 3T3-l1 adipocytes by using ultra-fast microscopy. Mol Biol Cell 12:129-41, 2001

40. Tsakiridis T, Tong P, Matthews B, Tsiani E, Bilan PJ, Klip A, Downey GP: Role of the actin cytoskeleton in insulin action. Microsc Res Tech 47:79-92, 1999

41. Chiang SH, Baumann CA, Kanzaki M, Thurmond DC, Watson RT, Neudauer CL, Macara IG, Pessin JE, Saltiel AR: Insulin-stimulated GLUT4 translocation requires the CAP-dependent activation of TC10. Nature 410:944-8, 2001

42. Amano M, Fukata Y, Kaibuchi K: Regulation and functions of Rho-associated kinase. Exp Cell Res 261:44-51, 2000

43. Wettschureck N, Offermanns S: Rho/Rho-kinase mediated signaling in physiology and pathophysiology. J Mol Med 80:629-38, 2002

44. Shibata H, Mukai H, Inagaki Y, Homma Y, Kimura K, Kaibuchi K, Narumiya S, Ono Y: Characterization of the interaction between RhoA and the amino-terminal region of PKN. FEBS Lett 385:221-4,
1996

45. Madaule $\mathrm{P}$, Eda M, Watanabe N, Fujisawa K, Matsuoka T, Bito H, Ishizaki T, Narumiya S: Role of citron kinase as a target of the small GTPase Rho in cytokinesis. Nature 394:491-4, 1998

46. Reid T, Furuyashiki T, Ishizaki T, Watanabe G, Watanabe N, Fujisawa K, Morii N, Madaule P, Narumiya S: Rhotekin, a new putative target for Rho bearing homology to a serine/threonine kinase, PKN, and rhophilin in the rho-binding domain. J Biol Chem 271:13556-60, 1996

47. Watanabe G, Saito $Y$, Madaule $P$, Ishizaki T, Fujisawa K, Morii N, Mukai H, Ono Y, Kakizuka A, Narumiya S: Protein kinase $N \quad(P K N)$ and $P K N$-related protein rhophilin as targets of small GTPase Rho. Science 271:645-8, 1996

48. Ohan N, Agazie Y, Cummings C, Booth R, Bayaa M, Liu XJ: RHO-associated protein kinase alpha potentiates insulin-induced MAP kinase activation in Xenopus oocytes. J Cell Sci 112 (Pt 13):2177-84, 1999

49. Zick Y: Insulin resistance: a phosphorylation-based uncoupling of insulin signaling. Trends Cell Biol 11:437-41, 2001

50. Aguirre V, Uchida T, Yenush L, Davis R, White MF: The c-Jun NH(2)-terminal kinase promotes insulin resistance during association with insulin receptor substrate-1 and phosphorylation of Ser(307). J Biol Chem 275:9047-54, 2000

51. De Fea K, Roth RA: Protein kinase $C$ modulation of insulin receptor substrate-1 tyrosine phosphorylation requires serine 612. Biochemistry 36:12939-47, 1997

52. Greene MW, Morrice N, Garofalo RS, Roth RA: Modulation of human insulin receptor substrate-1 tyrosine phosphorylation by protein kinase Cdelta. Biochem J 378:105-16, 2004

53. Moeschel K, Beck A, Weigert C, Lammers R, Kalbacher H, Voelter W, Schleicher ED,Haring HU, Lehmann R: Protein kinase C-zeta-induced phosphorylation of Ser318 in insulin receptor substrate-1 (IRS-1) attenuates the interaction with the insulin receptor and the tyrosine phosphorylation of IRS-1. J Biol Chem 279:25157-63, 2004

54. LiuYF, Paz K, Herschkovitz A, Alt A, Tennenbaum T, Sampson SR, Ohba M, Kuroki T, LeRoith D, Zick Y: Insulin stimulates PKCzeta -mediated phosphorylation 
of insulin receptor substrate-1 (IRS-1). A self -attenuated mechanism to negatively regulate the function of IRS proteins. J Biol Chem 276:14459-65, 2001

55. Ozes ON, Akca H, Mayo LD, Gustin JA, Maehama T, Dixon JE, Donner DB: A phosphatidylinositol 3-kinase/Akt/mTOR pathway mediates and PTEN antagonizes tumor necrosis factor inhibition of insulin signaling through insulin receptor substrate-1. Proc Natl Acad Sci U S A 98:4640-5, 2001

56. De Fea K, Roth RA: Modulation of insulin receptor substrate-1 tyrosine phosphorylation and function by mitogen-activated protein kinase. $J$ Biol Chem 272:31400-6, 1997

57. Jakobsen SN, Hardie DG, Morrice N, Tornqvist HE: 5'-AMP-activated protein kinase phosphorylates IRS-1 on Ser-789 in mouse C2C12 myotubes in response to 5-aminoimidazole-4-carboxamide riboside. $J$ Biol
Chem 276:46912-6, 2001

58. Um SH, Frigerio F, Watanabe M, Picard F, Joaquin M, Sticker M, Fumagalli S, Allegrini PR, Kozma SC, Auwerx J, Thomas G: Absence of S6K1 protects against age- and diet-induced obesity while enhancing insulin sensitivity. Nature 431:200-5, 2004

59. Qiao LY, Zhande R, Jetton TL, Zhou G, Sun XJ: In vivo phosphorylation of insulin receptor substrate 1 at serine 789 by a novel serine kinase in insulin -resistant rodents. J Biol Chem 277:26530-9, 2002

60. White MF: IRS proteins and the common path to diabetes. Am J Physiol Endocrinol Metab 283:E413-22, 2002

61. Kanda T, Wakino S, Homma K, Yoshioka K, Tatematsu S, Hasegawa K, Takamatsu I, Sugano N, Hayashi K, Saruta T: Rho-kinase as a molecular target for insulin resistance and hypertension. Faseb $J$ 20:169-71, 2006 